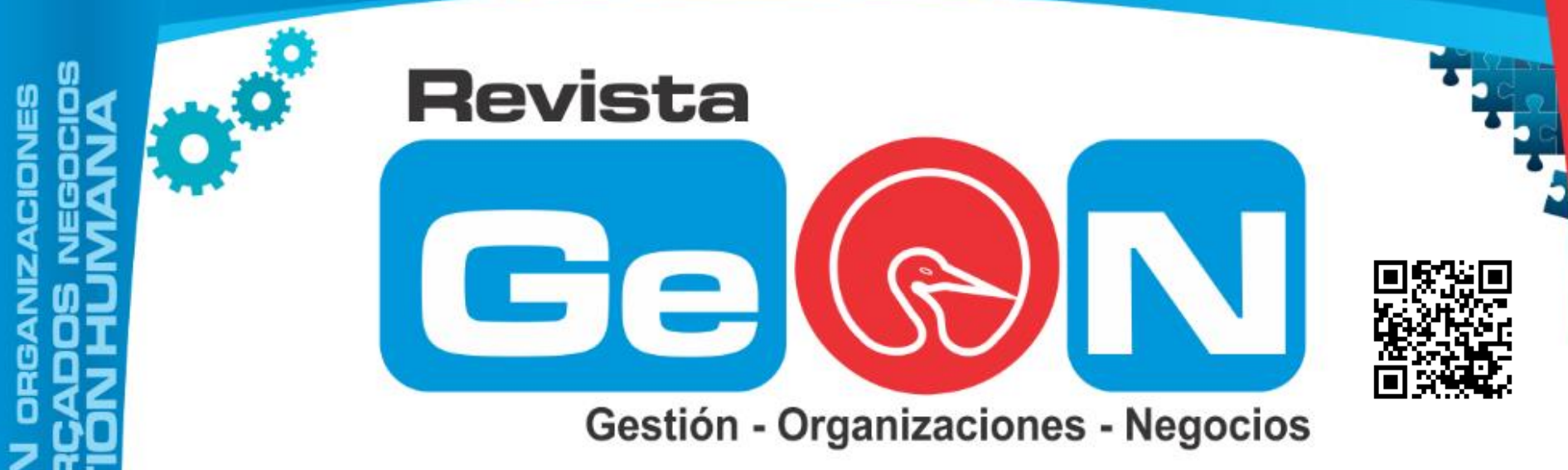

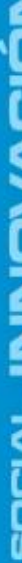

(1)

ISEN 204E - 3970

Volumen 11 Número 0 Diciembre Jullo 14

Revista Electrónica de la Facultad de Ciencias Económicas de la Universidad de los Llanos

$4 \leq 1$

$3<$

$2 \frac{1}{2} \frac{1}{4}$
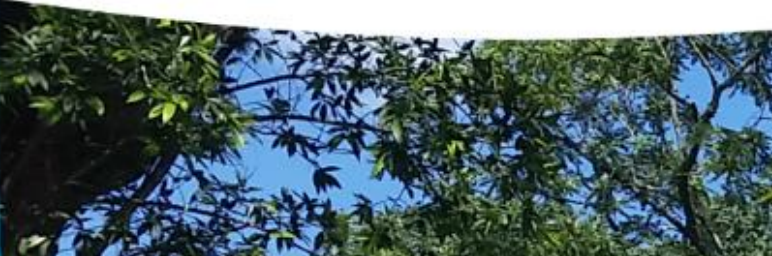

(1)

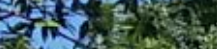

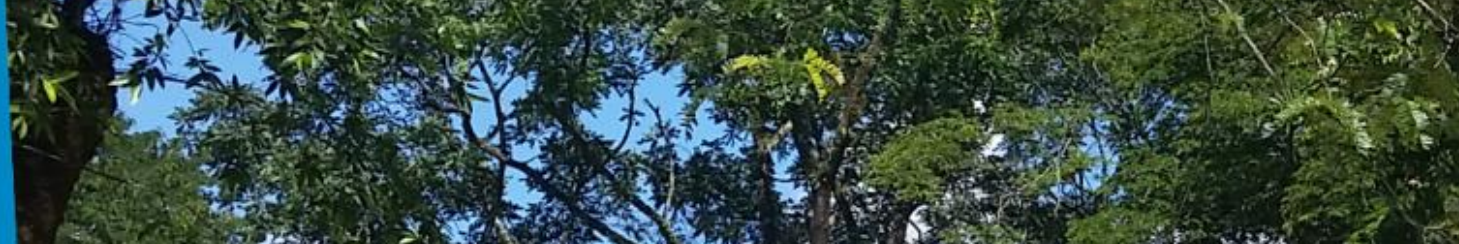

다

(11) को

$2 \frac{1}{0}$

i c

iii $\frac{2}{11}$ if

in

402

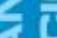

40

20 III

iㅣㄴ

12

10

10

ii 1

U1

$2 \frac{1}{2}$

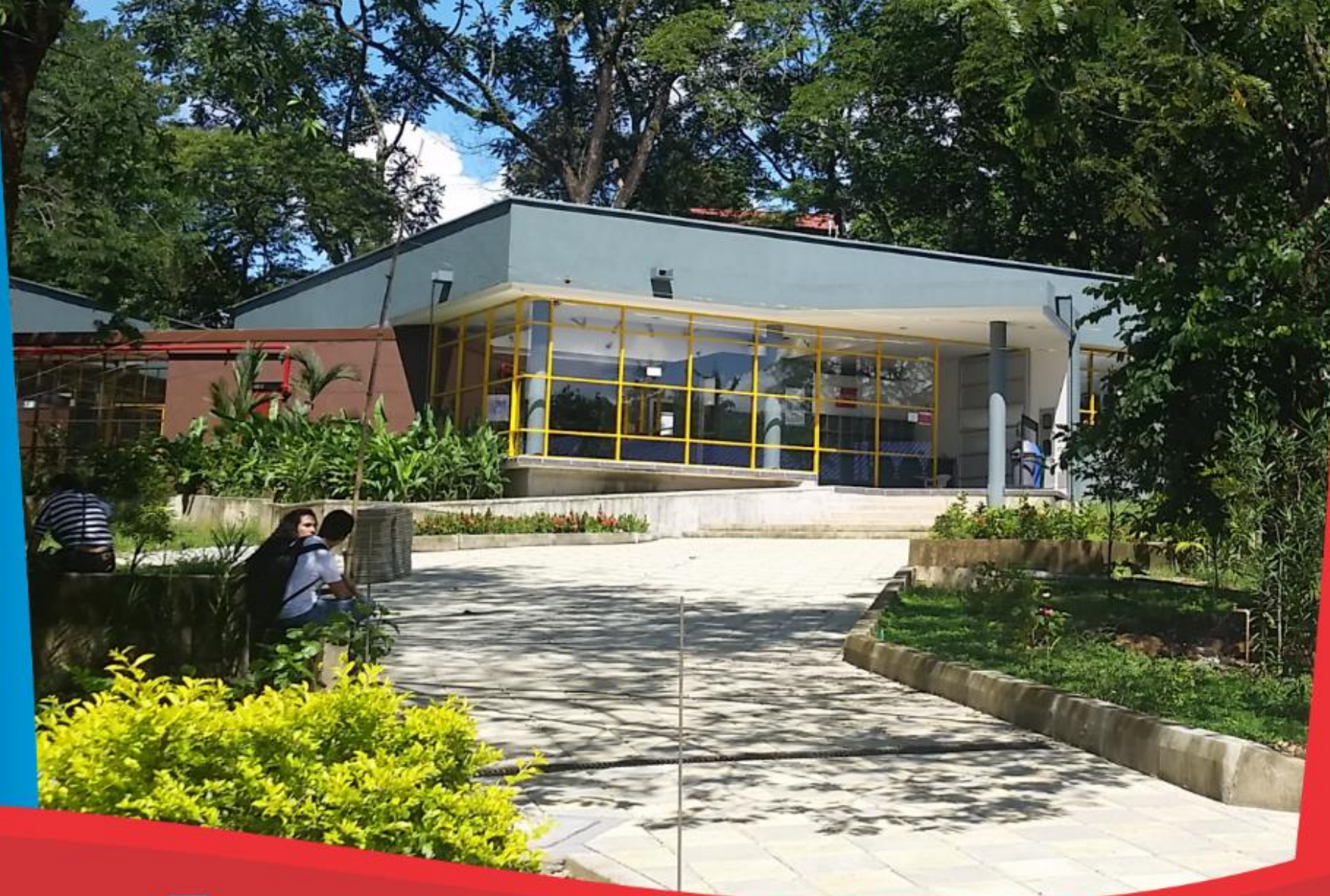

I/revistaGEON @RevistaGeon

http://revistageon.unillanos.edu.co 


\section{EMPLOYER BRANDING - Por Juan Pablo Giraldo}

Juan Pablo Giraldo Palacio

Estudiante de Administración de Empresas

Universidad de los Llanos

@ juangiraldop

La única parte donde el "éxito" aparece antes que el "trabajo" en el diccionario. Vidal Sasoon.

\section{RESUMEN}

La dirección de los recursos humanos se convierte en una actividad fundamental dentro del proceso administrativo que se ejecuta en cada organización, siendo pieza clave para el desarrollo y aplicación de estrategias que le permitan a la organización sobrevivir y competir en un determinado entorno. En las últimas décadas se ha evidenciado un claro cambio en la concepción sobre la importancia y relevancia del talento humano para las organizaciones; de tal forma, que muchas empresas han cambiado su filosofía y visión acerca de la manera de gestionar a las personas para que se alineen con la misión, objetivos y metas de cada una, dando como resultado a un sinfín de herramientas y teorías modernas que se enfocan a la manera de gestionar y motivar el talento humano dentro de las empresas. El Employer Branding es una herramienta para la gestión del talento humano; el cual permite una fuerte alineación entre los colaboradores y la organización, tomando como elemento fundamental de la estrategia el posicionamiento de la marca dentro del mercado laboral; para tal logro se utiliza como vehículo a los clientes internos o colaboradores.

\section{SUMMARY}

The management of human resources becomes a fundamental activity within the administrative process that runs on each organization, being key to the development and implementation of strategies that will enable the organization to survive and compete in an environment. In the last decades have shown a clear shift in the conception of the importance and relevance of human talent for organizations, in such a way that many companies have changed their philosophy and vision about how to manage people so that they align with the mission, objectives and goals of each, resulting in a host of tools and modern theories that focus on how to manage and motivate human talent within companies. The Employer Branding is a tool for the management of human talent which allows a strong alignment between employees and the organization, taking as a fundamental element of the strategy of the brand positioning in the labor market, for such achievement is used as a vehicle internal customers or collaborators

\section{LA IMPORTANCIA DE LA MOTIVACIÓN DEL TALENTO HUMANO}

Anteriormente se ha explicado la importancia que tiene el talento humano para las organizaciones en la era moderna y la manera como se desarrolla e implementa el Employer Branding para la gestión del talento humano cuyo fin es el posicionamiento de la empresa en un mercado como competitiva y fuente de reclutamiento de los mejores perfiles; así mismo, es necesario conocer como contribuye la motivación a tal logro y cuál es la plataforma estratégica que debe tener una empresa antes de implementar el Employer Branding.

La motivación es un proceso multifacético que con implicaciones individuales, administrativas y organizacionales. No solo es lo que el empleado muestra, sino todo un conjunto de aspectos ambientales que rodea al puesto de trabajo lo cual hace que un individuo actué y se comporte de una determinada manera dentro de la organización. (Rojas, 2004). Si bien la motivación en la organización se puede presentar de diferente manera; es claro que la empresa debe establecer ambientes de trabajo adecuados que permitan a los individuos desarrollarse íntegramente; de tal forma, que contribuyan al buen funcionamiento de la empresa. Este ambiente laboral en el cual se desenvuelve el individuo está compuesto por una serie de factores que van desde el tipo de contratación hasta la manera de como el individuo se desvincula de la misma.

Muchas organizaciones invierten cantidades de capital en el desarrollo de estrategias enfocadas a mejorar la cultura corporativa a través de un elemento fundamental como es la motivación de los individuos, ya que los resultados son relativamente beneficiosos tanto para las empresas como los colaboradores. Estas estrategias se enfocan en aspectos claves de la organización como salarios, establecimiento de un sistema de recompensas, planes de carrera y a un más importante un plan de entrenamiento y capacitación que le permita no solo al individuo fortalecerse profesionalmente sino 
ascender dentro de la misma. Toda esta plataforma se sustenta en la búsqueda de la motivación como factor clave para que el individuo se apropie de los objetivos estratégicos y ayude a la contribución del éxito de la compañía.

Por consiguiente, para poder establecer una estrategia de Employer Branding la organización debe tener definida una plataforma que permita desarrollar al individuo en un ambiente laboral que lo motive a ejecutar cualquier actividad pensando siempre en el bienestar para la organización, de tal forma; que la empresa se convierte en un sello o referencia del individuo ante cualquier decisión.

\section{RE-IMAGINA!}

El proceso de establecer una estrategia como el Employer Branding no es una tarea fácil; requiere de esfuerzos tanto económicos como personales, a fin de que se convierta en reto para muchas organizaciones que quieren ser líderes en el mercado. Este proceso requiere de innovación en procesos, en el estilo de liderazgo y más aún en la mentalidad de cómo se percibe la organización, este cambio requiere de una organización que "re-imagine" la manera de hacer las cosas, de concebir sus colaboradores y de cómo perciben los colaboradores la empresa; en sí es un proceso análogo en el cual la mentalidad y percepción es la que más cambia.

Re-imaginar como elemento fundamental de la estrategia Employer se sustenta en una serie de condiciones del ambiente que propicien cambios mentales en los colaboradores; (Peters, 2006) "conseguir que se hagan las cosas" no es cuestión de "poder" formal o de "rango" oficial. Es, en ultimo termino, una cuestión de..., PASIÓN, IMAGINACIÓN Y PERSISTENCIA.

$\mathrm{Y}$ es precisamente convertir hacer algo por funciones, en hacer algo por iniciativa. Esta iniciativa en el talento humano es el resultado de un proceso de transformación en la mentalidad de los individuos y al estar cambiando la mentalidad de los individuos directamente cambiara el modelo de cultura corporativa; es simple como cuando una vez se tira la ficha de dómino el efecto alcanza a todos.

Muchos autores sustentan que cambiar la cultura organizacional no es una tarea fácil, y que requiere de mucho tiempo y esfuerzo o aun mas muchas estrategias se convierten en fracaso al intentar transformar la cultura de una organización; y realmente no están fuera de contexto al establecer tal opinión, pues muchos casos lo han demostrado; pero surge una pregunta ¿a qué trabajador no le gustaría sentirse bien en su puesto de trabajo? ¿Que cada día se vuelva más competitivo? ¿Y que como resultados de sus iniciativas y esfuerzo obtenga más beneficios económicos? Ante tal plataforma que propicie esto los resultados serán asombrosos a fin de que la organización se convierta en un atractivo para personas que buscan el cambio, la superación y reconocimiento.

Dentro del proceso de re-imaginar en una organización se necesitan líderes o personas que cuenten con la capacidad de influir en otros colaborados con el objetivo de crear una red que cada día tenga más y más seguidores. Es necesario recordar que la estructura de cualquier organización atribuye unas condiciones especiales; por ejemplo el jefe tiene la capacidad y poder de atribuir y asignar tareas; pero también de liderar. ¿Pero cómo se lideraría dentro de un proceso de Employer Branding? (Peters, 2006)"la meta del trabajo del jefe debe ser esta: ¡conseguir que la gente... mucha, mucha gente... inicie, y trabaje después en proyectos WOW! ¡Proyectos por los que quieran ser recordados!" Por consiguiente el jefe o las personas que tengan a cargo el poder de dirigir deben tener esta capacidad única para liderar un proceso de Employer Branding dentro de las organizaciones, como así mismo influir positivamente dentro de los colaboradores. Todo esto transformándose en el conjunto de acciones que contribuyen a crear una plataforma adecuada para la implementación exitosa de una organización Employer Branding con personal altamente capacitado contribuyendo a un tipo de publicidad innovadora y capaz de posicionar a la empresa en un lugar privilegiado.

\section{EMPLOYER BRANDING}

Anteriormente se ha examinado los diferentes factores que contribuyen al éxito en la aplicación de una estrategia como el Employer Branding; resaltando aspectos puntuales como la cultura, la innovación y la motivación. Estos factores como ya se han mencionado anteriormente son pieza clave para las organizaciones y ante cualquier cambio son los más afectados. El Employer Branding se presenta como una de las más herramientas modernas en la gestión del recurso humano, el marketing y la competitividad de las organizaciones. "Employer 
Branding es la estrategia e iniciativas que una empresa adopta para lograr que su identidad corporativa tenga unos atributos asociados, y que sea percibida como atractiva por los profesionales. Es el sistema de gestión de la marca de la empresa cuyo objetivo es atraer, motivar y retener los "talentos". Se trata de construir una reputación de buen empleador, tanto para los empleados actuales como futuros. Pero además de la retención del talento, se busca que exista una mayor satisfacción, compromiso e identificación de los empleados con su compañía, ya que serán la piedra angular sobre la que se asienta la satisfacción de los clientes. En este sentido, una compañía verdaderamente orientada al cliente no puede ser una compañía que no esté orientada a las personas". (Dennis, 2012)

De acuerdo a esta definición, se identifican una serie de factores que son muy importantes para el buen desempeño de una organización y el éxito de la aplicación de una estrategia como esta. Al presentar la organización como una marca atractiva en un mercado laboral para poder atraer al personal más calificado se está desarrollando una serie de estrategias internas enfocadas a crear el mejor ambiente de trabajo; estrategias como ascensos, buenos salarios, beneficios, capacitación, entrenamiento entre otras.

La marca de una organización es una pieza fundamental para lograr la captación de clientes, pues en ella está implícita toda la organización; por ende se convierte en uno de los activos más valiosos de la empresa, así mismo esta se posiciona en la mente del consumidor ya sea por calidad, precio, diferenciación $\mathrm{u}$ otras características que se convierte en fortaleza competitiva de la empresa.

LA MARCA. El Employer utiliza estos dos factores claves para lograr ser una estrategia de alto impacto, pues la marca conocida y posicionada en el consumidor ahora es llevada por el cliente interno como máximo valor de atracción con el cual se identifica, posicionándola no solo en el cliente sino también en el mercado laboral.

Una vez ya identificada la marca como pieza fundamental dentro de la estrategia de Employer Branding es necesario conocer cuáles son los componentes esenciales que debe poseer esta para poder ser una imagen representativa para el grupo de colaboradores y los clientes. Los valores son fundamentales pues ellos deben ser el soporte social de la marca, de manera que estos son aceptados primero por los colabores y en segunda instancia se identifican con los clientes o segmentos de mercados; el posicionamiento es factor clave de éxito pues desde la empresa se debe definir como se quiere que se perciba la marca en el público y los colaboradores; por tanto el diseño, mensaje e impacto debe ser una actividad propia de los estrategas de la organización. La personalidad y atributos de la marca son muy importantes pues estos son los que trasmiten el mensaje a los clientes y colaboradores.

\section{LA ESTRATEGIA EN MARCHA.}

Poner en marcha una estrategia de Employer Branding requiere desarrollar una serie de acciones que permita lograr la instauración exitosa de la estrategia en la organización; pues es de vital importancia que la empresa conozca cuales son las fortalezas y debilidades de la marca en el mercado o en la mentalidad de los consumidores.

El posicionamiento es de mucha importancia pues determinar cómo está situada la marca en un mercado no en el carácter económico sino en la mentalidad permitiendo determinar el grado de penetración o impacto que se debe realizar al lanzar la estrategia; además permite obtener cual es la diferencia entre la situación real de percepción de la marca y la deseada.

se debe realizar un detallado análisis sobre las prácticas del mercado en esta materia; es decir, examinar si esta estrategia se desarrolla por la competencia en el mismo segmento de mercado.

es necesario que la organización determine concretamente cuales son los pilares esenciales que soportaran la imagen de la compañía; ya que a partir de esto se dirigirá la estrategia tanto externa para los clientes e interna para los colaboradores.

Establecer las herramientas para la determinación de la imagen interna; es decir, programas, planes, políticas e incentivos que ofrece la organización a sus colaboradores.

Identificar el mensaje o la idea central que llevara la marca, la promesa o compromiso que refleja la organización con el clima laboral, los clientes y la organización.

Todos estos pasos para poner en marcha una estrategia de Employer Branding le permiten a la 
empresa tener unas bases concretas en información tanto interna como externa que es utilizada en la generación y construcción de la estrategia como tal para la organización; si la empresa conoce de sus fortalezas y amenazas como de sus debilidades y oportunidades tendrá la certeza de desarrollar una adecuada estrategia para un adecuado personal.

\section{EL EMPLOYER BRANDING Y EL MARKETING}

Inicialmente se hablo acerca de la relación que existe entre el Employer Branding y el marketing en la exitosa implementación y de esta herramienta como medio de posicionamiento.

El marketing en las organizaciones se presenta como una herramienta eficaz que permite a las organizaciones lograr penetrar en un mercado utilizando estrategias publicitarias, de precios, merchandising, innovaciones, canales de distribución entre otras. De tal forma, que presenta una gran variedad de estrategias que pueden ser beneficiosas; el Employer Branding se apoya en el marketing a través del posicionamiento; llevando a establecer una estrategia que tiene como objetivo comunicar sobre la cultura corporativa, el bienestar, los ambientes de trabajo y las relaciones laborales presentando a la organización de manera indirecta en un mercado laboral cuya voz de publicidad son los clientes y trabajadores.

En consecuencia, es evidente que la manera de cómo se comporta el mercado laboral y el talento humano ha cambiado sustancialmente en la época moderna; pues el individuo posee una gran necesidad de superación y realización buscando los mejores entornos. "El momento actual, con nuevos perfiles de profesionales, exige una nueva actitud de la organización con un completo conocimiento y respeto por cada uno de los miembros de la empresa, por cada una de las Personas.

Las empresas deben preocuparse por lo que es realmente importante para sus Personas y, únicamente entonces, podrán responder con una proposición de valor desarrollada entorno a sus necesidades profesionales y personales" (Andrés, 2010). Por consiguiente; el Employer Branding responde a las necesidades de este tipo de personal creando las condiciones necesarias para poder tener éxito una organización.

\section{CONCLUSIONES}

La gestión estratégica del talento humano se convierte en una herramienta fundamental para lograr el desarrollo exitoso de las estrategias y que la organización responda de manera eficiente a los cambios generados en el entorno; siendo así el talento humano uno de los recursos más importantes para las organizaciones estas utilizan un conjunto de herramientas encaminadas a la motivación, integración, beneficio y clima laboral entre otros; que le permita a los colaboradores desarrollar plenamente sus capacidades y habilidades competitivas. La motivación juega un papel muy importante dentro del clima laboral de cualquier empresa, pues de ella depende la aptitud de nuestros colaboradores dentro del ambiente laboral, una motivación adecuada no solo permite que el individuo se motive a realizar sus funciones sino que también permite obtener clientes satisfechos; ya que toda estrategia que desarrolle una organización tanto interna como externa incidirá en los clientes.

Las personas motivadas realizan no solo sus funciones por obligación sino también las realizan por iniciativa propia, proponen y desarrollan ideas beneficiosas para su lugar de trabajo y organización; cuando una empresa posee un grado de motivación adecuado permite que el individuo se alinee estratégicamente con la misión, visión y objetivos corporativos. La estrategia de Employer Branding sin duda alguna es una herramienta moderna que se ajusta a las necesidades actuales del talento humano y permite que la organización sea más competitiva en los mercados; esta estrategia no solo se convierte en la apuesta por lograr cumplir económicamente dentro de la actividad propia de la empresa, sino como una herramienta que permite la transformación sustancial de las empresas a un estado en el cual su capital humano puede representar mayor valor que sus activos; no se trata de establecer una empresa dependiente de las exigencias de los colaboradores, pero si una organización que establece un balance entre la importancia del talento humano y la importancia de la generación de resultados positivos para seguir compitiendo.

Employer Branding es sin duda alguna una estrategia que resulta exitosa dependiendo del tipo y edad de la organización; pues bien tener un equipo de colaboradores altamente calificados requiere de recursos suficientes y de estrategias con un costo mayor al que generalmente desarrolla la industria. 
Una estrategia exitosa permite a la organización ser la empresa preferida entre el mercado laboral; obteniendo así los mejores colaboradores de un sector específico, este situación privilegiada en el mercado se logra a través del establecimiento de una marca como referencia, sello y motivo de orgullo para cual los diferentes colaboradores o clientes.

\section{BIBLIOGRAFÍA}

Rojas F. (2004). Motivación y comportamiento en la organización. Recuperado de http://www.degerencia.com/articulos.php?artid=513

Peter T. (2006) Re-imagina!: la excelencia empresarial en una era perturbadora. Madrid España. Pearson.

Peter T. (2006) Re-imagina!: la excelencia empresarial en una era perturbadora. Madrid España. Pearson.

Denis C. (2013) un enfoque dinámico del Employer Branding. Recuperado de http://www.tatumglobal.com/portal/index.php?option $=$ com_content\&view=article\&id=159:enfoquedinamico-employer-branding \&catid=25:espacios-deaprendizaje \&Itemid=3

Primo D \& de Andrés. (2013) se innovadoRH claves del marketing aplicadas a la gestión de personas. Recuperado de http://www.tatumglobal.com/portal/index.php?option $=$ com_content $\&$ view $=$ article $\& i d=127$ :nuevaestructura-funcionrrhh\&catid $=24$ :personas $\&$ Itemid $=3$ 